\title{
PROBLEMAS COM O NOMINALISMO DE CLASSE
}

\author{
Valdetonio Pereira de Alencar ${ }^{1}$ \\ Universidade Federal do Cariri (UFCA) \\ (D) https://orcid.org/0000-0001-8192-9396
}

\section{RESUMO:}

Neste artigo, analiso o Nominalismo de Classe como uma solução para o problema dos universais. Inicialmente, apresento a forma como entendo o problema dos universais. Em um segundo momento, caracterizo de maneira geral o Nominalismo de Classe. Neste ponto, indico as características gerais dessa posição. Posteriormente, analiso as posições de Quinton e Lewis. Além de apresentar as suas posições, mostro como eles tentam se livrar de algumas críticas clássicas. Defendo que esses autores não são bem sucedidos e que, portanto, a teoria deles apresenta sérios problemas. Por último, discuto dois tópicos críticos ao Nominalismo de Classe: o problema das relações e a questão da economia ontológica. Apresento razões para essa posição não fornecer uma análise adequada para as relações. Por fim, defendo que, apesar dessa posição ser uma teoria nominalista, ela não é tão econômica de um ponto de vista ontológico como se poderia esperar de uma forma de Nominalimo.

PALAVRAS-CHAVE: Nominalismo de Classe; Classes Naturais; Lewis.

\section{TROUBLES WITH CLASS NOMINALISM}

\begin{abstract}
:
In this paper, I discuss the Class Nominalism as a solution to the problem of universals. At first, I attempt a clarification of the problem of universals. Secondly, I provide the definition of Class Nominalism. Later, I discuss the theses of Lewis and Quinton and I analyze how they try to get rid of some classic critiques. I argue that they are not successful and, therefore, their theory has serious problems. Finally, I discuss two critical topics to Class Nominalism: the problem of relations and the problem of ontological economy. I think the class nominalism does not provide an adequate analysis of relations. Finally, I argue that this theory is not

\footnotetext{
${ }^{1}$ Doutor em Filosofia pela Universidade Federal do Rio de Janeiro (UFRJ), Rio de Janeiro - Brasil. Professor da Universidade Federal do Cariri (UFCA), Ceará - Brasil. E-mail: valdetonio_alencar@yahoo.com.br
} 
economic (from an ontological point of view). There are realistic and nominalists theories more economical than Class Nominalism.

KEY WORDS: Class Nominalism; Natural Classes; Lewis.

\section{0 problema dos universais e o Nominalismo de Classe}

O problema dos universais é considerado um problema ontológico, pois diz respeito a que tipos de entidades existem. Duas categorias são tradicionalmente indicadas como candidatas a categorias ontológicas fundamentais: particular e universal. Particulares são entidades não repetíveis, que podem ser concretas e abstratas. Universais são entidades repetíveis e, normalmente, consideradas abstratas.

Especificamente, o problema dos universais consiste em saber se universais constituem entidades não redutíveis na constituição ontológica do mundo. Notoriamente, há duas respostas principais a esse problema: o Realismo e o Nominalismo. O Realismo consiste na tese de que universais são entidades não redutíveis à descrição ontológica do mundo. $\mathrm{O}$ Nominalismo, de uma forma geral, considera que a noção de propriedade pode ser analisada sem nos comprometermos com universais.

Ao definir particular e universal, uma segunda distinção ontológica emerge: a distinção entre entidades abstratas e concretas. Entidades concretas possuem poder causal e localização espaço-temporal, enquanto entidades abstratas não possuem poder causal nem localização espaçotemporal. Essa distinção (bem como a distinção entre particulares e universais) parece-me útil para discernir as posições envolvidas no debate. Para o nominalista mais extremo ${ }^{2}$, existe a palavra "cachorro", existem vários objetos particulares que são cachorros. Não existe nada além do que particulares concretos. Não existe outra entidade no mundo que corresponda à palavra "cachorro". Para discernir esse tipo de Nominalismo de outros como o Nominalismo de Classe, não é suficiente afirmar que esse Nominalismo radical admite apenas particulares. Afinal, o Nominalismo de Classe também admite apenas particulares. Mas há uma diferença fundamental. Para o Nominalismo de Classe, além de existirem vários objetos particulares que são cachorros, existe a classe dos cachorros. Pareceme que o Nominalismo de Classe introduz um novo tipo de entidade na sua ontologia, pois não existem apenas os particulares acerca dos quais um determinado predicado aplica-se. Existe uma entidade (particular, mas abstrata) que corresponde a esse predicado, que é uma classe. O Nominalismo de Classe implica, assim, a existência de particulares concretos e particulares abstratos (classes).

\footnotetext{
2 Armstrong (1978, p. 11) denominaria essa postura de "Nominalismo de Predicado".
} 
Em primeiro lugar, apresentarei uma caracterização geral do Nominalismo de Classe. Nesse momento, discutirei as motivações para a formulação dessa postura. Em segundo lugar, tratarei do Nominalismo de Classe de Quinton, que introduziu a noção de classe natural nessa posição. Em terceiro lugar, analisarei uma forma mais sofisticada de Nominalismo de Classe desenvolvida por David Lewis. Por último, discutirei o comprometimento ontológico do Nominalismo de Classe. Em particular, discutirei a noção de economia ontológica.

\section{Nominalismo de Classe Ingênuo}

Wolterstorff (1970, p. 173) afirma que a identificação de universais com classes é antiga e já estaria presente na época de Abelardo. Esse, ao apresentar e discutir algumas posições acerca do problema dos universais, afirma o seguinte:

Some people take the universal thing as consisting only in a collection of a several things. They do not at all call Socrates and Plato by themselves a "species"; rather they call all men collected together the "species" man, and all animals taken together the "genus" animal, and so on for other cases. (ABELARDO, 1994, p. 34).

Não é claro, a partir dessa citação, se o Nominalismo que Abelardo comenta é um Nominalismo de Classe. Esse Nominalismo parece explicar universais não a partir de classes, mas sim de coleções de objetos. Esse tipo de Nominalismo é conhecido como "Nominalismo Mereológico" (ARMSTRONG 1978, pp. 34-35). A distinção entre Nominalismo de Classe e Nominalismo Mereológico é importante para entender qual é o comprometimento ontológico do Nominalismo de Classe. Segundo o Nominalismo Mereológico, temos o termo predicativo "F", existem os vários F's e existe a soma mereológica dos F's, que é também uma entidade particular. A soma mereológica dos F's ocupa a função de universal nesse tipo de Nominalismo. Diferentemente de universais, somas mereológicas não acrescentam nada (de um ponto de vista ontológico) em relação aos particulares que as compõem, pois são tão particulares e abstratas quanto os particulares que as compõem. O Nominalismo de Classe postula, além dos F's, a classe dos F's que desempenha a função de universal nesse Nominalismo. Diferentemente da soma mereológica dos F's, a classe dos F's não é ontologicamente redutível aos F's. Tomem-se duas classes quaisquer: $\{a, b\}$ e $\{\{a\}, b\}$. Apesar de serem duas classes distintas, temos que elas são compostas dos mesmos particulares concretos (SHOTTENKIRK 2009, pp. 23-24). Assim, o Nominalismo de Classe acrescenta um tipo de entidade em sua ontologia, não redutível a particulares concretos. Enquanto isso, o Nominalismo Mereológico não 
acrescenta nenhum tipo de entidade em nossa ontologia, pois fusões mereológicas são tão concretas quantos os particulares, que as compõem.

Os dois principais autores que defenderam o Nominalismo de Classe foram Quinton e David Lewis. Antes de tratar destes autores, apresento, nesta seção, as características gerais de qualquer Nominalismo de Classe. Denomino o Nominalismo de Classe apresentado nesta seção de "Nominalismo de Classe ingênuo" por dois motivos. Em primeiro lugar, parece-me ser uma posição que apresenta muitos problemas. Em segundo lugar, não conheço nenhum autor que tenha defendido essa forma de Nominalismo de Classe. Para apresentar essa posição, tomarei como base a caracterização da mesma feita por Armstrong (1978).

De um ponto de vista epistemológico, o Nominalismo de Classe é uma solução objetivista para o problema dos universais, pois classes possuem uma existência independente da mente e da linguagem humana. $\mathrm{O}$ fato de um particular $a$ possuir uma propriedade $F$ deve ser analisado como $a$ sendo um elemento da classe que possui como elementos os particulares que são $F$. E, para explicar relações n-árias, o Nominalismo de Classe poderá utilizar classes de n-uplas ordenadas. Como classes ordenadas podem ser definidas através de classes não ordenadas, tem-se que a análise do nominalista de classe permanece padronizada para propriedades e relações.

À primeira vista, parece que não se ganha muito em utilizar classes em vez de universais para se explicar propriedades, pois universais e classes são entidades abstratas. Um dos motivos que fez com que nominalistas rejeitassem a existência de universais estava na desconfiança acerca de entidades abstratas. Além disso, se pensarmos a partir do princípio de economia ontológica, é defensável que o Realismo seja uma teoria mais econômica. Se for possível construir toda a realidade a partir da categoria de universal, então teríamos que o Realismo compromete-se com apenas um tipo de entidade, tanto na distinção concreto/abstrato quanto na distinção particular/universal (O'LEARY-HAWTHORNE, J. e COVER, 1998). O Nominalismo de Classe compromete-se com um tipo de entidade segundo a distinção particular/universal, mas compromete-se com dois tipos de entidades segundo a distinção concreto/abstrato, isto é, o Nominalismo de Classe compromete-se com particulares concretos e particulares abstratos. Se o Nominalismo de Classe e o Realismo possuírem o mesmo valor explanatório, então o Realismo (pelo menos do tipo acima especificado) deveria ser a melhor teoria.

A utilização de classes para explanar propriedades, contudo, parece justificar-se por vários motivos. A teoria de classes parece se encontrar em melhor estado do que a teoria dos universais. Classes possuem condições de identidade determinadas, universais não. Não é muito clara a relação entre particulares e universais. Na história da Filosofia, recorreu-se muitas vezes a metáforas, como a da participação, para explicar a relação entre particulares 
e universais. Para o Nominalismo de Classe, essa relação é expressa pelo fato de particulares pertencerem a determinadas classes. Além disso, o Nominalismo de Classe parece resolver problemas que o Realismo resolve, mas que outras formas de Nominalismo não conseguem resolver. Entre esses problemas, podemos apontar o da referência abstrata dos termos gerais (LOUX 2006, pp. 26-30) e o de como explicar a predicação (LOUX 2006, pp. 21-26). Isso parece tornar essa solução Nominalista muita atraente.

No entanto, há muitas críticas contra ao Nominalismo de Classe (ARMSTRONG 1978, cap. 4). A crítica clássica consiste no problema das propriedades co-extensionais. Caso você não tenha universais em sua ontologia e você explique propriedades através de classes, surge o problema de que você pode ter propriedades diferentes que são instanciadas pelos mesmos particulares. $\mathrm{O}$ exemplo clássico é o das propriedades ser criatura com rins e ser criatura com coração. Temos, nesse caso, duas propriedades que estabelecem a mesma classe. Parece, assim, que a nossa fala sobre propriedades não pode ser substituída por uma fala sobre classes.

Outro problema que Armstrong (1978, pp. 42-43) levanta concerne à questão da causalidade:

The object acts in virtue of the fact that it has a mass of four kilograms. But it is that individual four-kilogram object that acts. The other four-kilogram things in the universe seem to be irrelevant. However, if a class analysis of what it is to be four kilograms in mass is correct, then the whole class of tokens should be relevant: The four-kilo property of the individual is constituted by its class membership. But in fact, though, the only thing relevant is the thing that actually acts. This suggests that any class account of properties is unsatisfactory. (ARMSTRONG 1989, p. 28).

Em síntese, Armstrong reclama que a explicação de propriedades através de classes não consegue explicar o poder causal de um objeto. Seria estranho, entretanto, se classes conseguissem ser uma boa explicação do poder que um objeto tem de agir sobre nós a partir de suas propriedades, pois classes não possuem poder causal.

Outro problema de aceitar propriedades como classes é que, apesar de toda propriedade estabelecer uma classe, nem toda classe estabelece uma propriedade. Parecem existir classes mais interessantes do que outras. Além disso, a explicação do porquê de um objeto possuir uma propriedade devese ao fato de ele pertencer a uma classe segundo o nominalista de classe. Intuitivamente, parece ser o contrário. Um objeto $a$ pertence à classe dos F's se $a$ possuir a propriedade $F$. O Nominalismo de Classe parece ser incapaz de explicar esses problemas. 
Wolterstorff (1970, pp. 173-181) faz várias críticas a quem pretende identificar propriedades com classes ${ }^{3}$. Uma que me parece interessante diz respeito às condições de identidade das classes. Todos os elementos de uma classe pertencem à mesma necessariamente, isto é, dada uma classe $A=\{1,2,3,4\}$, se 4 não pertencesse a $A$, então teríamos outra classe. $O$ mesmo não ocorre com propriedades. Mesmo que a classe das coisas brancas fosse diferente, ainda assim teríamos a mesma propriedade. Ora, propriedades, portanto, possuem condições de identidade diferentes de classes. Por isso, aquelas não podem ser identificadas com estas.

\section{Nominalismo de Classe de Quinton}

Outro argumento interessante contra o Nominalismo de Classe ingênuo é fornecido por Quinton. Contra essa identificação de propriedades com classes, ele apresenta o seguinte problema:

For any collection of $\mathrm{n}$ things there are $2^{n}-1$ non-empty classes of varying size from 1 to $n$ members in which they can be arranged: the class composed of all $n$ of them, the $n$ classes of $n$ -1 of them and so on. 7 things can be arranged in over 100 classes, 10 things in over a thousand, 20 things in over a million. But can 20 things really have a million common properties or respects of resemblance? (QUINTON 1973, p. 262).

A reclamação de Quinton é que você pode formar classes cujos elementos não possuem nenhuma semelhança entre si. Nenhuma propriedade corresponde a essas classes. Uma resposta a esse problema seria afirmar que haveria uma propriedade em comum nesses casos. Por exemplo, tome-se a classe cujos elementos sejam: 2 , João e a Lua. Nesse caso, ter-seia a seguinte propriedade: ser 2-João-Lua. Sem dúvida, de um ponto vista gramatical, "ser 2-João-Lua" pode ser um predicado em uma determinada linguagem. Contudo, esse predicado não parece representar uma propriedade. No máximo, poderíamos afirmar que ele representa uma propriedade disjuntiva ser 2 ou ser João ou ser Lua. O problema de propriedades disjuntivas é que elas não são necessárias na nossa descrição de mundo, pois são redutíveis a propriedades não disjuntivas. Além disso, o problema posto por Quinton permanece, pois a classe em questão teria elementos que não possuem nenhuma semelhança entre si.

Apesar de sua crítica ao Nominalismo de Classe ingênuo, Quinton ainda pretende identificar propriedades com classes. No entanto, ele pensa que apenas certo tipo de classe é capaz de ocupar a função das propriedades.

\footnotetext{
${ }^{3} \mathrm{Na}$ verdade, Wolterstorff critica quem identifica predicados com classes. Mas as críticas são igualmente aplicáveis à identificação de propriedades com classes.
} 
Dentre as classes, Quinton destaca as classes naturais. Ele define classes naturais utilizando as noções de semelhança e propriedade:

That are natural classes is entailed by the existence of reapplicable predicates but does not entail it. For there to be a reapplicable predicate the naturalness of some class, a common property or a resemblance, has to be noticed and to be named. (QUINTON 1973, p. 263).

Segundo Quinton, todas as classes naturais devem ter, pelo menos um elemento, o que implica que a classe vazia não é uma classe natural. Isso impossibilita a existência de propriedades não instanciadas.

Não fica claro, no ponto de vista de Quinton, o que é uma propriedade, pois ele não pode definir propriedade como sendo qualquer classe. Segundo ele, propriedades são classes naturais. Mas, para definir classe natural, ele utiliza a noção de propriedade. Não há uma redução completa da teoria de propriedades para teoria de classes, pois o fato de um objeto $a$ possuir a propriedade $F$ não é redutível a $a$ pertencer à classe dos F's. Na explanação do fato de um objeto $a$ possuir a propriedade $F$, o autor utiliza a noção de propriedade que torna os objetos da classe dos F's semelhantes. Isso parece caracterizar a explanação de Quinton como circular.

$\mathrm{O}$ autor reivindica que a distinção ente classes naturais e classes arbitrárias é ontológica (QUINTON, 1957, p. 45). Contudo, essa tese não parece tão clara. Haack (1969, p. 316) e Armstrong (1978, p. 40) discordam da mesma. O que define uma classe como natural, para Quinton, consiste no fato dos objetos dessa classe possuírem uma propriedade em comum. Contudo, propriedade não é uma categoria ontológica. É uma noção préanalítica, que precisa ser analisada. No mundo, o que temos são classes. E não há nada na própria classe, de um ponto de vista ontológico, que indique que uma classe seja natural. Assim, o Nominalismo de Quinton não parece conseguir analisar propriedades de maneira completa. Aparentemente, Quinton precisa de uma noção primitiva (não analisável) de propriedade para sustentar que classes são naturais. Defender que a noção de propriedade é primitiva significa aproximar o Nominalismo de Classe do Nominalismo de Avestruz.

Além disso, o Nominalismo de Classe Natural de Quinton também não escapa nem do problema da co-extensionalidade, nem das críticas de Armstrong e nem da crítica de Wolterstorff. Uma teoria que pretende resolver alguns desses problemas é a de Lewis, que será tratada na próxima seção.

\section{Nominalismo de Classe David Lewis}


David Lewis apresenta-nos uma teoria de propriedades composta por três elementos:

1. A teoria de classes;

2. Uma teoria realista acerca dos mundos possíveis e

3. A distinção entre propriedades escassas ou naturais e abundantes.

Veremos que a grande novidade do Nominalismo de Classe de Lewis ${ }^{4}$ é a utilização de sua teoria acerca dos mundos possíveis para solucionar o problema dos universais. A análise de Lewis sobre propriedades visa mostrar a vantagem de utilizar a sua teoria sobre mundos possíveis. Ele destaca algumas vantagens: sua análise de propriedade é puramente extensional, não recorre a universais e soluciona alguns problemas que o Nominalismo de Classe em geral não conseguia resolver.

Segundo Lewis, como nós quantificamos sobre propriedades, nós precisamos de entidades para fazer a função de propriedades. A maneira mais simples é entender propriedades como a classe de suas instâncias e utilizar a teoria de classes para explicar o fato de $a$ possuir a propriedade $F$. Contudo, ele está consciente de que esse ponto de vista possui problemas. Duas propriedades diferentes podem ser co-extensivas. E, se uma propriedade fosse uma classe, então as instâncias dessa propriedade teriam que ter essa propriedade necessariamente, pois uma classe é determinada por seus elementos. Com elementos diferentes, temos classes diferentes. Mas, com instâncias diferentes, não temos propriedades diferentes. As condições de identidade de uma propriedade são diferentes das condições de identidade de uma classe. De forma sucinta, esse é o problema posto por Wolterstorff.

A solução para esses problemas encontra-se no Realismo Modal de Lewis. Ele afirma que propriedades são classes de possibilia (LEWIS 1983, p. 209). Assim, a propriedade $F$ é identificada com a classe das instâncias dessa propriedade em todos os mundos possíveis. Lewis pode recorrer a isso por ter uma concepção realista acerca dos mundos possíveis. Os mundos de Lewis são tão concretos quanto o mundo atual. Eles existem da mesma maneira que o mundo atual, e os mundos possíveis são isolados espaçotemporalmente. Por isso, não há relações causais entre mundos possíveis. As propriedades ser criatura com rins e ser criatura com coração possuem a mesma extensão apenas no mundo atual. Evidentemente, há mundos possíveis nos quais uma criatura pode ter coração sem ter rins. Essas duas propriedades não são co-extensivas ao longo dos mundos possíveis. A análise de propriedades como classes de possibilia também pode ser utilizada para tratar de relações (LEWIS 1986, p. 52). Uma instância de uma relação binária seria um par ordenado. Assim, a relação seria uma classe de todas as suas instâncias ao longo dos mundos possíveis.

\footnotetext{
${ }^{4}$ Pode ser alvo de crítica defender que Lewis seja um nominalista de classe. De qualquer modo, é uma interpretação razoável a partir do texto New work for a theory of universals: "I already have at my disposal a theory of properties as classes of possibilia" (1983, p 209).
} 
Essa estratégia parece resolver o problema de Wolterstorff. Segundo Lewis:

\begin{abstract}
It is a mistake to say that if a property were a set, then it would have its instances - its members - essentially, and therefore it never could be contingent whether something has or lacks it. Consider the property of being a talking donkey, which I say is the set of all talking donkeys throughout the worlds. The full membership of this set does not vary from world to world. What does vary from world to world is the subset we get by restricting ourselves to the world in question. (LEWIS 1986, p.51).
\end{abstract}

A classe dos F's é a classe dos objetos que são $F$ ao longo dos mundos possíveis. Essa classe não pode ser diferente do que de fato é. Nesse aspecto, o problema de Wolterstorff nem se levanta, pois os mundos de Lewis esgotam todas as possibilidades (ARMSTRONG 1989, p.27).

Resta, contudo, observar que o problema da co-extensionalidade não está completamente resolvido. A princípio, poderíamos ter um caso de duas propriedades diferentes que são necessariamente co-extensionais. O exemplo clássico é o caso das propriedades ser triângulo e ser trilátero. Claramente, ser triângulo e ser trilátero são propriedades diferentes. Mas como elas são necessariamente co-extensionais, então essas duas propriedades constituiriam a mesma classe de possibilia. A solução de Lewis para esse problema envolve a noção de propriedade estruturada:

This conception demands that properties should be structured. If we want to watch up properties with the meanings of linguistic expressions that have syntactic structure, then we want to give the properties themselves some king of quasisyntatic structure. (LEWIS 1986, p. 56).

Lewis tenta mostrar que termos gerais distintos são utilizados para referir-se a propriedades co-extensionais através de estruturas sintática diferentes. Tomemos o exemplo utilizado por Lewis: ser triângulo e ser trilátero. Sejam A e S relações não estruturadas, tal que A é a relação ser ângulo de, e $\mathrm{S}$ é a relação ser um lado de. Seja $\mathrm{T}$ uma relação não estruturada de ordem superior entre uma propriedade não estruturada $F$ e uma relação não estruturada $\mathrm{G}$ se, e somente se, $\mathrm{F}$ é a propriedade com a qual exatamente três coisas têm a relação G. Assim, T é definida a partir do par ordenado $(\mathrm{F}, \mathrm{G})$. A partir dessas definições, Lewis pode definir as propriedades ser triângulo e ser trilátero. Ser triângulo é a propriedade de ser algo com a qual exatamente três coisas possuem a relação de ser ângulo de. Ser trilátero é a propriedade de ser algo com a qual exatamente três coisas possuem a relação de ser lado de. Tendo-se essas definições, pode-se definir as propriedades ser triângulo e ser trilátero através dos respectivos pares ordenados: $(\mathrm{T}, \mathrm{A})$ e $(\mathrm{T}, \mathrm{S})$. Temos que, nessa definição estruturada, ser 
triângulo e ser trilátero seriam redefinidas através de relações que não são necessariamente co-extensionais, pois A e S são classes diferentes ${ }^{5}$ (LEWIS 1986, p. 56). Essa definição estruturada de propriedade é compatível com a definição não estruturada, pois propriedades continuam a ser classes.

Outro aspecto importante do Nominalismo de David Lewis é a distinção entre propriedades abundantes e propriedades escassas. Essa distinção faz-nos lembrar da distinção entre classes naturais e classes não naturais feita por Quinton. Segundo Lewis, propriedades abundantes são extrínsecas e não qualitativas. $\mathrm{O}$ fato de dois objetos compartilharem a mesma propriedade abundante não implica que esses objetos sejam semelhantes. Quando Lewis fala que propriedades são classes, ele está referindo-se a propriedades abundantes. "In fact, the properties are as abundant as the sets themselves, because for any set whatever, there is the property of belong to that set." (LEWIS 1986, p. 60) ${ }^{6}$. Propriedades abundantes não capturam as semelhanças objetivas nem possuem poderes causais. Quase todas as propriedades são causalmente irrelevantes (LEWIS 1983, p. 211). Propriedades escassas são bem diferentes das abundantes. Elas são intrínsecas e naturais. Os objetos que compartilham propriedades escassas apresentam similaridade qualitativa. Exemplos de propriedades escassas são propriedades físicas fundamentais, como ter carga elétrica.

Lewis parece fazer essa distinção por um motivo semelhante ao de Quinton: existem algumas classes mais interessantes do que outras. Essas classes mais interessantes são as estabelecidas por propriedades escassas. Parece que as propriedades abundantes não são problemáticas, pois o fato de um objeto $a$ possuir uma propriedade abundante $F$ é redutível a $a$ pertencer à classe dos $\mathrm{F}$ 's. $\mathrm{O}$ mesmo não ocorre com as propriedades escassas. $\mathrm{O}$ fato de um objeto $a$ possuir uma propriedade escassa $F$ não é redutível a $a$ pertencer à classe dos F's. Além disso, o fato de $a$ ser $F$ implica que eu estou afirmando algo qualitativo sobre $a$. E isso não é redutível a $a$ pertencer à classe dos F's. Parece que Armstrong está certo: $a$ pertence à classe dos F's porque $a$ possui a propriedade escassa $F$, e não o contrário.

Outra crítica de Armstrong não respondida por esse Nominalismo concerne ao poder causal das propriedades escassas. Como classes não possuem poder causal, então não teríamos como explicar o poder causal das propriedades escassas. Um aspecto estranho da teoria de propriedades de Lewis concerne à relação entre a sua concepção de mundo possível e sua teoria de propriedades. As classes não pertencem a nenhum mundo possível, pois mundos possíveis são entidades concretas. Ora, se propriedades são

\footnotetext{
${ }^{5}$ De uma forma semelhante, Lewis (1986, pp. 56-57) utiliza essa definição de propriedades estruturadas para tratar de relações estruturadas.

${ }^{6}$ Segundo a definição de propriedade abundante, pertencer à classe vazia seria uma propriedade abundante. Como pertencer à classe vazia é uma classe de possibilia, então seria uma propriedade não instanciada.
} 
classes e classes não pertencem a nenhum mundo, então propriedades não pertencem a nenhum mundo.

Tenho dúvidas se a solução de Lewis para o problema de Wolterstorff é de fato uma boa solução. Wolterstorff levanta o seguinte ponto: as condições de identidade de uma classe são diferentes das condições de identidade de uma propriedade. Ele levantou esse problema a partir da observação de que a classe dos seres humanos poderia ser diferente do que de fato é. Isso tornaria a classe dos seres humanos outra classe, devido ao princípio de extensionalidade da teoria de classes. Apesar disso, a propriedade ser humano continuaria a ser a mesma. Assim, Wolterstorff espera ter mostrado que as condições de identidade de uma classe são diferentes das condições de identidade de uma propriedade. Lewis responde a essa crítica, mostrando que o exemplo não ocorre. A classe dos seres humanos é a classe dos objetos que são humanos ao longo dos mundos possíveis. Essa classe não pode ser diferente do que de fato é. O problema de Wolterstorff parece só ter aparecido quando pensamos que propriedades são classes compostas por instâncias apenas do mundo atual. De fato, a resposta de Lewis é satisfatória em mostrar que o exemplo não consegue apontar que as condições de identidade de uma classe são diferentes das condições de identidade de uma propriedade. O problema de Wolterstorff, contudo, é mais geral do que esse exemplo. O problema é sobre condições de identidade. Tome-se a propriedade escassa $F$. Essa propriedade corresponde a certa classe de possibilia. Toda classe tem sua identidade determinada por seus elementos. O fato da classe dos F's não poder ser diferente do que é não implica que $\mathrm{F}$ não tenha as condições de identidade que classes normalmente possuem. Pelo contrário, a classe dos F's continua a ser governada pelo princípio da extensionalidade. $\mathrm{O}$ mesmo não ocorre com propriedades. $\mathrm{O}$ fato de um objeto ter ou não a propriedade escassa $F$ parece ser irrelevante para a identidade de $F$. Em que sentido João, que é humano, faz parte das condições de identidade da propriedade ser humano? Intuitivamente, a propriedade ser humano não depende de João para ser a propriedade que é. Por isso, o Nominalismo de Lewis não supera o problema levantado por Wolterstorff.

\section{Um problema acerca das relações}

Como foi mencionado anteriormente, o nominalista de classe tratará relações como conjuntos de pares ordenados. Assim como toda a teoria de conjunto, a noção de par ordenado é bem estabelecida. E pode ser definida através de conjuntos não-ordenados. A primeira definição bem sucedida de par ordenado foi feita por Nobert Wiener em 1914 (ENDERTON, 1977, p. 36):

$$
\text { (1) }(\mathrm{x}, \mathrm{y})=\{\{\{\mathrm{x}\}, \varnothing\},\{\{\mathrm{y}\}\}\}
$$


No entanto, uma definição mais simples foi dada por Kuratowski em 1921. Esta definição é, geralmente, a mais usada nos dias de hoje:

(2) $(x, y)=\{\{x\},\{x, y\}\}$

Ambas as definições (assim como outras) satisfazem o critério de identidade de um par ordenado:

(3) $(\mathrm{u}, \mathrm{v})=(\mathrm{x}, \mathrm{y})$ se e somente se $\mathrm{u}=\mathrm{x}$ e $\mathrm{v}=\mathrm{y}$

Essas definições são particularmente úteis para o nominalista de classe, pois essa posição estaria comprometida com particulares concretos e classes (particulares abstratos). Ao tratar pares ordenados como classes não ordenadas, o nominalista de classe permite que sua análise das propriedades e relações mantenha-se constante, isto é, o comprometimento ontológico seja estabelecido com o mesmo tipo de entidade em ambos os casos. Apesar das definições (1) e (2) satisfazerem o critério de identidade de um par ordenado, ainda seria possível apontar problemas para o nominalista de classe. O primeiro problema concerne a certo caráter convencional dessas definições (RODRIGUEZ-PEREYRA, 2002, p. 59). Tome-se a seguinte relação binária: x ama y. No Nominalismo de Classe, essa relação seria analisada da seguinte maneira:

(4) $(x$ ama y) $=\{(a, b),(b, a),(c, d),(d, c) \ldots\}$

A relação $\mathrm{x}$ ama $\mathrm{y}$ consiste no conjunto formado pelos pares ordenados assinalados acima. Como existem pelo menos duas definições de par ordenado, poderíamos traduzir (4) de duas maneiras:

(5) $(x$ ama y) $=\{\{\{\{a\}, \varnothing\},\{\{b\}\}\}, \quad\{\{\{b\}, \varnothing\},\{\{a\}\}\}$, $\{\{\{c\}, \varnothing\},\{\{d\}\}\},\{\{\{d\}, \varnothing\},\{\{c\}\}\}, \ldots\}$

(6) $(x$ ama y) $=\{\{\{a\},\{a, b\}\},\{\{b\},\{a, b\}\},\{\{c\},\{c, d\}\}$, $\{\{\mathrm{d}\},\{\mathrm{c}, \mathrm{d}\}\}, \ldots\}$

Para a teoria de conjuntos, não há problema em termos duas definições. O importante é convencionar uma delas como padrão. Para a disciplina Ontologia, não é tão simples assim. A investigação ontológica consiste, de maneira prioritária, em determinar o que existe. Ora, a relação $\mathrm{x}$ ama y é analisada de duas maneiras diferentes em (5) e (6). A rigor, dependendo de qual análise queremos escolher, teremos análises ontológicas diferentes. Não há nenhum critério ontológico para escolher entre essas análises. Ambas são corretas, mas apenas uma delas deveria ser verdadeira. Ao meu ver, isso indica que a análise do nominalista de classe das relações é artificial e ad hoc.

Essa análise apresenta um segundo problema. Vamos supor que (6) representa a correta análise das relações. Relações consistiriam ontologicamente em classes de classes. Propriedades unárias consistiriam em classes de objetos. Tome-se o seguinte ordenamento lógico de entidades:

0 - Objetos

1 - Propriedades e relações de primeira ordem

2 - Propriedades e relações de segunda ordem 
Os exemplos de propriedades e relações utilizados até aqui foram de propriedades de primeira ordem. Naturalmente, o Nominalismo de Classe teria que fornecer uma análise para propriedades e relações de ordem superior a 1. Tome-se a propriedade ser uma cor. Essa propriedade é de segunda ordem. Ela caracteriza propriedades de primeira ordem, como: ser azul, ser verde, etc. Propriedades de segunda ordem poderiam ser compreendidas como classes de classes segundo o Nominalismo de Classe. Assim, a propriedade de segunda ordem ser uma cor poderia ser analisada da seguinte maneira:

verdes,...$\}$

Ser um cor $=\{$ classe das coisas azuis, classe das coisas

De uma forma semelhante à feita acima, é possível estabelecer uma hierarquia de classes:

0 - objetos

1 - classes de objetos

2 - classes de classes de objetos

...

Seria interessante, para o nominalista de classes, que a hierarquia de propriedades e relações correspondesse de uma maneira perfeita à hierarquia de classes. Isso ocorre com as propriedades. Propriedades de primeira ordem serão analisadas como classes de objetos. Propriedades de segunda ordem serão analisadas como classes de classes de objetos, e assim por diante. O mesmo não ocorre com as relações. Relações de primeira ordem serão tratadas como classes de classes de objetos. Assim, ao se analisar propriedades de segunda ordem e relações de primeira ordem como classes, chega-se à conclusão de que ambas estão no mesmo nível de ordenamento ontológico. Isso fere uma intuição básica de que propriedades e relações de primeira ordem estão no mesmo nível ontológico, pois ambas caracterizam objetos. E que, portanto, relações de primeira ordem e propriedades de segunda ordem encontram-se em níveis ontológicos distintos.

\section{Nominalismo de Classe e o Princípio de Economia Ontológica}

Na Filosofia e nas Ciências é comum, por vezes, fazer uso de algum princípio de economia, seja conceitual, seja ontológico. No debate sobre o problema dos universais, o Nominalismo constitui a postura que levanta a bandeira da economia ontológica. A formulação desse princípio pode ser sumarizada no seguinte lema: não se deve multiplicar entidades sem necessidade. Eu não tenho como objetivo justificar esse princípio neste artigo, mas gostaria de elaborar melhor esse lema. Quando o nominalista defende que uma teoria mais econômica é melhor, ele está referindo-se não à quantidade de entidades de forma absoluta, mas sim à quantidade de tipos de entidades. Supostamente, quanto menos tipos de entidades, melhor para a sua teoria. Contudo, essa formulação ainda não é suficientemente desejável 
para o Nominalismo, pois seria possível nós termos formas de Realismo mais econômicas do que algumas formas de Nominalismo. Se o problema é apenas de quantidade de tipos de entidades, é provável que o realista pudesse utilizar o princípio de economia ontológica contra o nominalista. Se o realista puder construir toda a realidade utilizando apenas a categoria de universal (O'LEARY-HAWTHORNE, J. e COVER, 1998), então ele estaria comprometido com apenas um tipo de entidade. Ao tentar fazer o inventário do mundo através da categoria de particular concreto, o nominalista encontrar-se-ia em situação semelhante. Assim, do ponto de vista da economia ontológica, ambas as posturas estariam em situação semelhante.

Essa conclusão não é aceitável para os nominalistas, pois economia ontológica é uma motivação central dessa postura. O Nominalismo tradicional entende que particulares concretos formam uma categoria ontológica especial. Vejamos as seguintes passagens:

\footnotetext{
My awareness of the original coloured object is perceptual, but my awareness of the abstract universal redness cannot be since redness is not in space or time (QUINTON, 1973, p. 258).

For the main reason to believe in the existence of concrete particulars is not that they help to solve the Problem of Universals. On the contrary, that they exist is a presupposition of the Problem of Universals (RODRIGUEZ-PEREYRA, 2002, p. 211).
}

Quinton considera que particulares concretos são objetos dados à nossa percepção, diferentemente de universais cuja apreensão ocorreria de maneira misteriosa. Rodriguez-Pereyra considera que diferentemente dos universais - que são postulados de maneira ad hoc para solucionar o problema dos universais -, particulares não constituem entidades inferidas. Com base nessas observações, podemos formular a distinção entre entidades de acesso imediato e entidades inferidas (entidades introduzidas de maneira ad hoc). Ao defender o princípio de economia ontológica, é provável que o nominalista esteja referindo-se às entidades inferidas: não multiplique entidades inferidas sem necessidade. Como o Realismo postula universais para solucionar o problema dos universais, tem-se que essa postura seria idealmente menos econômica do que o Nominalismo Tradicional de uma forma geral. Resta, contudo, analisar o Nominalismo de Classe no que diz respeito à economia ontológica.

Analisemos a questão do comprometimento ontológico desse tipo de Nominalismo. O Nominalismo de Classe compromete-se com particulares concretos, classes (particulares abstratos) e possibilia (no caso da versão de Lewis). À primeira vista, classes e possibilia parecem ser entidades ad hoc. Classes foram introduzidas para substituir universais na Ontologia. E possibilia foram introduzidos para solucionar o problema da coextensionalidade. Rodriguez-Pereyra discorda que classes e possibilia sejam entidades ad hoc. 
Lewis (1986, p. 2) has emphasized, possibilia are of a kind with actual concrete particulars. Furthermore, the main reason to believe in possibilia is not that they help to solve the Problem of Universals in a Resemblance Nominalist way. They are not just postulated in order to solve the Problem of Universals; they do useful philosophical work elsewhere, notably in providing truth-conditions for modal discourse. Similarly with classes, ordered and unordered, which are a different kind of particulars and more controversial than concrete ones, but which we have independent reasons to accept. (RODRIGUEZ-PEREYRA, 2002, p. 212).

Possibilia são particulares concretos. Como eles são úteis para fornecer condições de verdade para o discurso modal, Rodriguez-Pereyra considera que possibilia não são introduzidos ad hoc. Apesar dessas entidades realizarem essa tarefa, existem teorias não realistas acerca dos mundo possíveis. Assim, a necessidade de fornecer condições de verdade para o discurso modal não constitui razão suficiente para aceitar possibilia. Porém ser nominalista de classe (ou nominalista de semelhança, no caso de Rodriguez-Pereyra) parece nos obrigar a ser realista acerca dos mundos possíveis e, consequentemente, aceitar possibilia na nossa ontologia. Por isso, consideramos que possibilia constituem entidades ad hoc. Do ponto de vista do Nominalismo de Classe, o problema da co-extensionalidade constitui o motivo para aceitar possibilia. Apesar de possibilia serem particulares concretos, eles não constituem entidades de acesso imediato. Nesse aspecto, o nominalista de classe introduz um terceiro tipo de entidade em sua ontologia: particulares concretos inferidos (introduzidos de maneira ad hoc).

Rodriguez-Pereyra defende que classes também não são entidades ad hoc. Tradicionalmente, classes ocupam uma função basilar na Matemática, pois a teoria de classes axiomatizada fornece elementos suficientes para o desenvolvimento daquela disciplina como um todo (ENDERTON, 1977, p. 11). Se a matemática envolver algum comprometimento ontológico, classes seriam naturais candidatas para fornecer a ontologia da Matemática. Estaríamos, assim, justificados em aceitar classes em nossa ontologia. Dado que existem classes, não parece haver problema em tomar algumas delas para exercerem a função das propriedades naturais.

Além das classes, temos elementos dessas classes. Assim, na hierarquia ontológica das classes temos o nível 0 , formado por objetos, mais precisamente: o conjunto de todos os objetos. Os níveis seguintes seriam formados a partir da aplicação da operação de potência $\mathrm{P}$ sobre um conjunto qualquer:

$$
\begin{aligned}
& 0-\text { conjunto dos objetos }=\mathrm{A} \\
& 1-\mathrm{P}(\mathrm{A})=\mathrm{V} 1 \\
& 2-\mathrm{P}(\mathrm{V} 1)=\mathrm{V} 2
\end{aligned}
$$


...

Apesar de uma teoria de classes poder ser construída dessa forma, não é necessário que seja assim. O conjunto dos átomos não serve para nenhum propósito matemático específico (ENDERTON, 1977, p. 9). Poderse-ia tomar o conjunto dos átomos como sendo o conjunto vazio. A hierarquia das classes seria formada apenas por conjuntos puros, sem necessidade de objetos externos.

$$
\begin{aligned}
& 0-\mathrm{A} 1=\varnothing \\
& 1-\mathrm{A} 2=\mathrm{P}(\mathrm{A} 1)=\{\varnothing\} \\
& 2-\mathrm{A} 3=\mathrm{P}(\mathrm{A} 2)=\{\varnothing,\{\varnothing\}\} \\
& 3-\mathrm{A} 4=\mathrm{P}(\mathrm{A} 3)=\{\varnothing,\{\varnothing\},\{\{\varnothing\}\},\{\varnothing,\{\varnothing\}\}\} \\
& \ldots
\end{aligned}
$$

Apesar de infinitas classes aparecerem nessa hierarquia, é evidente que as classes que interessam ao nominalista de classe não estão presentes, pois particulares concretos não compõem os átomos. Assim, mesmo que tenhamos que supor classes em nossa ontologia, não é necessário que tenhamos classes que satisfaçam as necessidades do nominalista de classe. Restaria ele argumentar que, como a teoria de classes irá implicar a existência de infinitas classes, não parece haver problema em acrescentar mais algumas (classes não puras) que sirvam para ocupar a função de propriedades naturais. Nesse caso, a justificativa para a introdução dessas classes não seria meramente matemática, mas sim para solucionar o problema dos universais. Isto é, as classes naturais constituiriam entidades ad hoc.

A última hierarquia de classes (cuja base é constituída apenas pelo conjunto vazio) é muito atrativa. Mesmo que não existisse nenhum particular concreto, toda a hierarquia estaria preservada. A Matemática, por conseguinte, não dependeria do mundo físico. Essas classes parecem ser verdadeiramente abstratas. As classes naturais, como toda classe que possui como elementos objetos espaço-temporais, apresentam alguns enigmas. Se classes possuem uma existência necessária, como elas podem ter como elementos objetos cuja existência é contingente? Se classes são particulares sem localização espaço-temporal (entidades abstratas), como podem ter elementos espaço-temporais (entidades concretas)? (OLIVER, 1993, p. 189190). Essas questões indicam que, além de serem ad hoc, classes naturais podem ser entidades com um caráter contraditório ${ }^{7}$.

Portanto, o realista poderia reclamar que o nominalista de classe também utiliza entidades $a d$ hoc. Aliás, ele utiliza dois tipos de entidades $a d$ hoc: possibilia e classes. Esse nominalista teve que explanar propriedades através de classes. Além disso, teve que postular possibilia para solucionar o

\footnotetext{
7 À primeira vista, universais também possuem um enigma que indica um caráter contraditório dessa noção: como o mesmo universal pode estar presente em diferentes particulares?
} 
problema da co-extensionalidade. Um dos motivos para se preferir classes é que elas são particulares e bem definidas. Contudo, poderíamos comparar as seguintes entidades: particulares concretos, classes e universais. Particulares concretos são, segundo o nominalista tradicional, ocorrências únicas na natureza (não repetíveis), possuem poder causal (pois são concretos) e são entidades de acesso imediato (não inferidas). Classes são entidades não repetíveis, abstratas e ad hoc. Universais são entidades repetíveis, abstratas e ad hoc. Se você comparar essas caracterizações, classes possuem mais semelhanças com universais do que com particulares concretos. Como os universais são entidades problemáticas, pois são repetíveis, abstratas e $a d$ hoc, e classes possuem algumas características que universais possuem, então classes talvez não sejam o caminho correto para o nominalista tentar explanar propriedades.

\section{Referências bibliográficas}

ABELARDO, Pedro. From the "glosses on Porphyry" in his logica 'ingredientbus'. In: SPADE, P. V. (ed.). Five Texts on the mediaeval problem of universals: Porphyry, Booethius, Abelard, Duns Scotus, Ockham. Introdução, tradução e notas: Paul Vincent Spade. Indianapolis/Cambridge: Hackett Publishing Company, 1994.

ARMSTRONG, David Malet. A theory of universals: volume I: nominalism and realism. Cambridge: Cambridge University Press, 1978.

Press, 1989.

Universals: an opinionated introduction. San Francisco: Westview

BENACERRAF, Paul. What numbers could not be. In: BENACERRAF, P. e PUTNAM, H. Philosophy of mathematics: selected readings. Cambridge: Cambridge University Press, 1983.

ENDERTON, Herbert. Elements of set theory. San Diego: Academic Press, 1977.

GOODMAN, Nelson e QUINE, Willard Von Orman. Steps toward a constructive nominalism. The Journal of Symbolic Logic. V. 12, No 4, pp. 105-122, dez. 1947.

HAACK, R. J. Natural and arbitrary classes. Australian Journal of Philosophy. Volume 47, No 3, pp. 307-321, 1969.

LEWIS, David. On the plurality of worlds. Oxford: Blackwell, 1986.

Nominalistic set theory. Nous. Volume 4, No 3, pp. 225-240, set.

1970

New work for a theory of universals (1983). In: KIM, J. e SOSA,

E. (Ed.). Metaphysics: an anthology. Oxford: Blackwell, 1999. p. 209-232.

LOUX, Michael. Metaphysics: a contemporary introduction. 3. ed. New York: Routledge, 2006.

O'LEARY-HAWTHORNE, John e COVER, Jan. A world of universals. 
Philosophical Studies, Volume 91, pp. 205-219, 1998.

OLIVER, Alex. Classes and Goodman's nominalism. Proceedings of the Aristotelian Society, New Series, Vol. 93, pp. 179-191, 1993.

QUINTON, Anthony. Properties and classes. Proceedings of the Aristotelian Society, New Series, Volume 58, pp. 33-58, 1957.

. The nature of things. London: Routledge and Kegan Paul, 1973

RODRIGUEZ-PEREYRA, Gonzalo. Resemblance nominalism: a solution to the problem of universals. Oxford: Oxford University Press, 2002.

SHOTTENKIRK, Dena. Nominalism and its aftermath: the philosophy of Nelson Goodman. New York: springer, 2009.

WOLTERSTORFF, Nicholas. On universals: an essay in ontology. Chicago: The University of Chicago Press, 1970. 